\title{
Die Wertigkeit konventioneller Röntgenaufnahmen des Beckens für die Erkennung eines Morbus Perthes 3 Monate nach durchgemachter Coxitis fugax
}

\section{The Value of Conventional Radiographs of the Pelvis in Detection of Perthes Disease 3 Months After an Episode of Acute Transient Synovitis}

\section{Autoren}

U. Lenoir ${ }^{1}$, T. Slongo ${ }^{1}$, E. Aghayev ${ }^{2}$, A. Joeris ${ }^{1}$

Institute

1 Department of Pediatric Surgery, Children's Hospital, Inselspital, University Hospital Bern, Bern, Switzerland

2 Institute of Social and Preventive Medicine, University of Bern, Bern, Switzerland

Schlüsselwörter

Coxitis fugax, klinischer Verlauf, Morbus Perthes, Verlaufsröntgen

Key words

transient synovitis, clinical course, Perthes disease, follow-up x-ray

Korrespondenzadresse

Ursina Lenoir

Department of Pediatric Surgery

Children's Hospital, Inselspital

University Hospital Bern

Freiburgstraße 4

3010 Bern

Switzerland

Tel.:+41/31/63292 23, Fax:+41/31/6329292

ursina.w@bluemail.ch

\section{ZUSAMMENFASSUNG}

Hintergrund Die häufigste Hüftgelenksaffektion beim Kind ist die Co-xitis fugax. Der im ähnlichen Altersspektrum auftretende Morbus Per-thes ist unter Umständen im Anfangsstadium nicht von einer Coxitis fugax zu unterscheiden. Aus diesem Grunde bekamen alle Kinder, die in unserer Klinik wegen einer Coxitis fugax behandelt wurden, nach 3 Monaten eine Röntgenkontrolle, um frühzeitig einen Morbus Perthes zu erkennen. Ziel dieser Studie war es, zu prüfen, ob alle Kinder mit einer Coxitis fugax-Episode ein Kontrollröntgen brauchen oder ob die Indika-tion dafür aufgrund des klinischen Verlaufes gestellt werden kann.
Patienten und Methode Retrospektive Analyse aller Kinder, die von 2004-2010 mit der Diagnose einer Coxitis fugax in unserer Klinik behandelt wurden. 198 Kinder mit der Diagnose einer Coxitis fugax wurden eingeschlossen. Ausgewertet wurden die radiologischen und klinischen Befunde bei Erstkonsultation und im Verlauf nach mindestens 3 Monaten. Ergebnisse Im Intervall zwischen Coxitis fugax und Verlaufskontrolle hatten 20 Kinder erneut Hüftbeschwerden (10,1\%). 16 davon hatten eine blande radiologische Verlaufsbildgebung, bei 4 Patienten ( $2 \%$ ) wurde ein Morbus Perthes diagnostiziert. Alle Kinder ohne Beschwerden im Verlauf hatten ein negatives Verlaufsröntgenbild (Sensitivität 0,2, Spezifität 1,0). Schlussfolgerung Eine Röntgenkontrolle nach Coxitis fugax erscheint nur dann notwendig, wenn im Intervall zwischen Coxitis fugax und Verlaufskontrolle nach 3 Monaten Beschwerden auftreten oder persistieren. Eine gute Anamnese und eine fachkundige klinische Nachkontrolle sind Voraussetzung, um zu entscheiden, ob eine radiologische Verlaufskontrolle nach 3 Monaten notwendig ist.

\section{ABSTRACT}

Background Transient synovitis is the most common hip joint disorder in children. Perthes disease occurs around the same age and may not be distinguishable to an episode of transient synovitis in the early stage. Therefore all children in our clinic with transient synovitis underwent a follow-up X-ray 3 months later to detect Perthes disease at an early stage. The aim of the study was to evaluate, if a follow-up X-ray is necessary for all children with suspected transient synovitis or if the clinical follow-up can lead to the indication for a follow-up X-ray.

Patients and Method Retrospective study including all children treated with the diagnosis of transient synovitis between 2004 and 2010.198 patients with the diagnosis of a transient synovitis were included. We analyzed the radiological and clinical findings initially and after at least 3 month follow-up.

Results In the time between the episode of transient synovitis and follow-up 20 children did not remain symptom-free (10.1\%). Of these patients 16 had a normal radiological follow-up and 4 (2\%) were diagnosed with Perthes disease. All children which remained symptom-free between the episode of transient synovitis and the follow-up had a negative follow-up X-ray (sensitivity 0.2 , specifity 1.0 ).

Conclusion A follow-up X-ray in detection of Perthes disease for children with transient synovitis appears to be necessary only if they have recurrent or persisting symptoms in the clinical course between the episode of transient synovitis and the 3-months-follow-up. A good patient history and an expert clinical follow-up examination are mandatory to decide whether a follow-up X-ray is needed. 


\section{Hintergrund}

Plötzlich auftretende Beschwerden des Hüftgelenks bei Kindern bedürfen einer genauen Abklärung, da je nach Erkrankung gravierende Langzeitschäden am Hüftgelenk resultieren können. Die häufigste Hüftaffektion, die bei Kindern im Alter von 4 bis 10 Jahren zu beobachten ist, stellt die Coxitis fugax (auch Hüftschnupfen, Coxitis simplex, transient synovitis, irritable hip, toxic coxitis genannt) dar [6]. Nach Landin ist sie mit einer durchschnittlichen jährlichen Inzidenz von $0,2 \%$ und einem akkumulierten Risiko von $3 \%$, mindestens eine Hüftschnupfen Episode zu erleiden, die häufigste Hüftgelenkserkrankung im Kindesalter [13]. Die Coxitis fugax tritt bei Knaben etwas mehr als doppelt so häufig auf als bei Mädchen $[4,7,13]$.

Die Coxitis fugax ist ein akuter, selbstlimitierender, nicht-infektiöser Erguss im Hüftgelenk, der ohne bleibende Schäden zu verursachen, wieder vollständig abheilt.

Es ist eine Ausschlussdiagnose, die nur gestellt wird, wenn anamnestisch, klinisch, laborchemisch und radiologisch andere Erkrankungen nicht festgestellt bzw. ausgeschlossen werden konnten $[13,17]$. Anamnestisch liegt vorausgehend häufig ein Infekt der oberen Atemwege oder des Magendarmtraktes vor. Die Kinder sind in einem guten Allgemeinzustand ohne hohes Fieber. Das Auftreten ist in der Regel akut. Klinisch zeigt sich eine Bewegungseinschränkung der Hüfte, eventuell ein Schonhinken. Laborchemisch findet sich keine oder nur eine minime Erhöhung der Entzündungsparameter, konventionell radiologisch ein Normalbefund und sonografisch allenfalls eine Synovialverdickung und ein Gelenkserguss.

Differenzialdiagnostisch muss vor allem die eitrige Coxitis ausgeschlossen werden, daneben Osteomyelitis, juvenile rheumatoi- de Arthritis, Lyme-Borreliose, epiphysäre Dysplasie und Epiphysiolysis capitis femoris $[3,4,8,11,13]$.

Eine weitaus weniger häufige, im gleichen Alterssegment auftretende sehr wichtige Differenzialdiagnose, ist der Morbus Perthes, eine aseptische Hüftkopfnekrose unbekannter Ursache, deren Verlauf sich häufig bis über mehrere Jahre hinzieht und zu bleibenden Hüftkopfdeformitäten mit Inkongruenz der gelenkbildenden Flächen mit daraus resultierender sekundärer Koxarthrose führen kann [12]. Der Morbus Perthes verläuft in bekannten Stadien mit jeweils typischen radiologischen Veränderungen des Femurkopfes: Initialstadium, Kondensationsphase, Fragmentationsphase, Reossifikationsstadium und Ausheilungsstadium. Er zeigt sehr unterschiedliche Verläufe, abhängig von verschiedenen Faktoren wie Ausdehnung und Lokalisation der Nekrose, Vorhandensein von radiologischen sogenannten „head-at-risk“-Zeichen (u. a. laterale Subluxation, laterale Kalzifikation, metaphysäre Beteiligung), sowie Alter des Patienten bei Erkrankung [10,12,19]. Knaben sind häufiger betroffen. Das Verhältnis Jungen zu Mädchen beträgt gemäß Literatur ungefähr 4:1 [7, 14].

In der Diagnostik der Coxitis fugax zeigt sich im Ultraschall fast immer ein Erguss des Hüftgelenks. Die konventionelle Röntgenaufnahme in 2 Ebenen ist normal ( $\triangleright \mathbf{A b} \mathbf{b} . \mathbf{1 a - c}$ ). In gleicher Art und Weise kann sich das Initialstadium eines Morbus Perthes mit Erguss (oder auch ohne) im Ultraschall und unauffälligem Befund im konventionellen Hüftröntgen darstellen, was die Unterscheidung zur Coxitis fugax in diesem Stadium unmöglich macht ( $>$ Abb. $2 \mathbf{2 a}-\mathbf{c}$ ) [12].

In der Vergangenheit wurde der mögliche Zusammenhang zwischen Coxitis fugax und Morbus Perthes ausführlich diskutiert. So wurde das Risiko des Auftretens eines Morbus Perthes nach einer
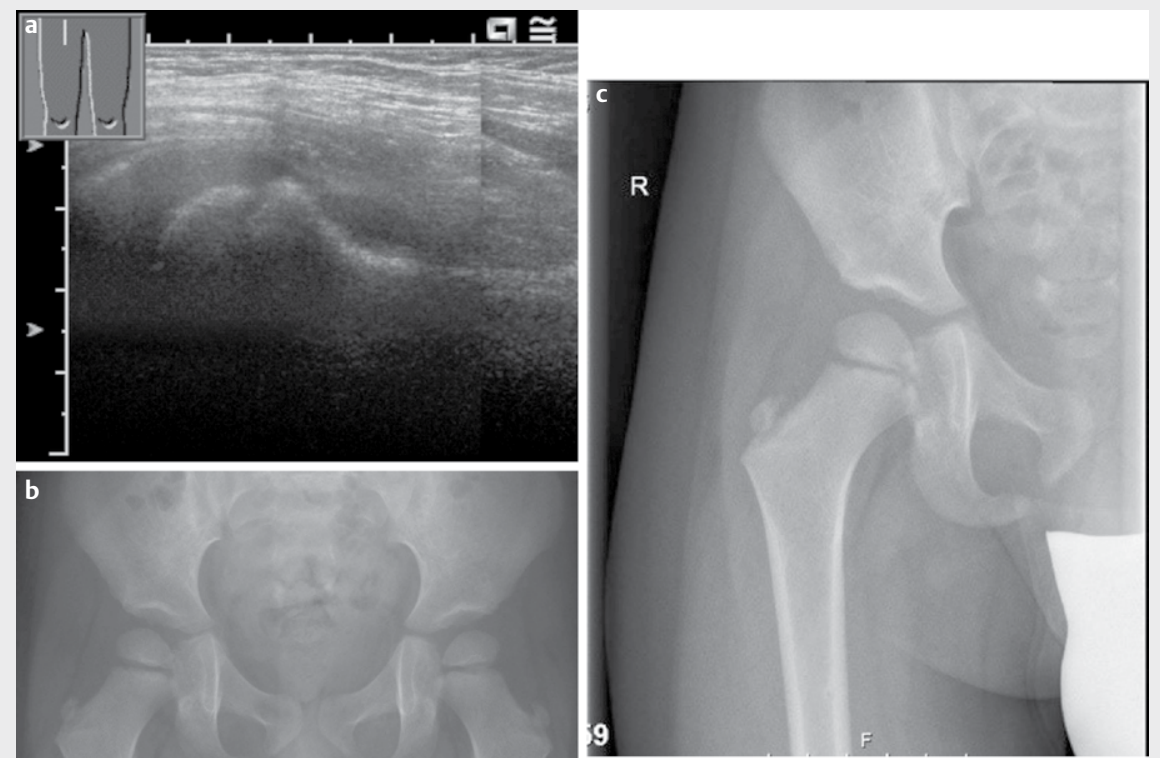

Abb. 1 a 4-jähriger Junge mit einem sonografisch nachgewiesenen Hüftgelenkserguss rechts im ventralen Longitudinalschnitt. b Unauffällige Röntgenübersichtsaufnahme des Beckens bei Erstpräsentation. Verdachtsdiagnose der Coxitis fugax bei klinisch eingeschränkter Hüftgelenksbeweglichkeit und gutem, afebrilem Allgemeinzustand. c Die anterior-posteriore Röntgenaufnahme des rechten Hüftgelenks desselben Patienten 3 Monate nach Auftreten der Hüftbeschwerden. Der Junge war zum Zeitpunkt der Nachkontrolle und im Intervall beschwerdefrei, sodass retrospektiv die Diagnose einer Coxitis fugax bestätigt werden konnte. 

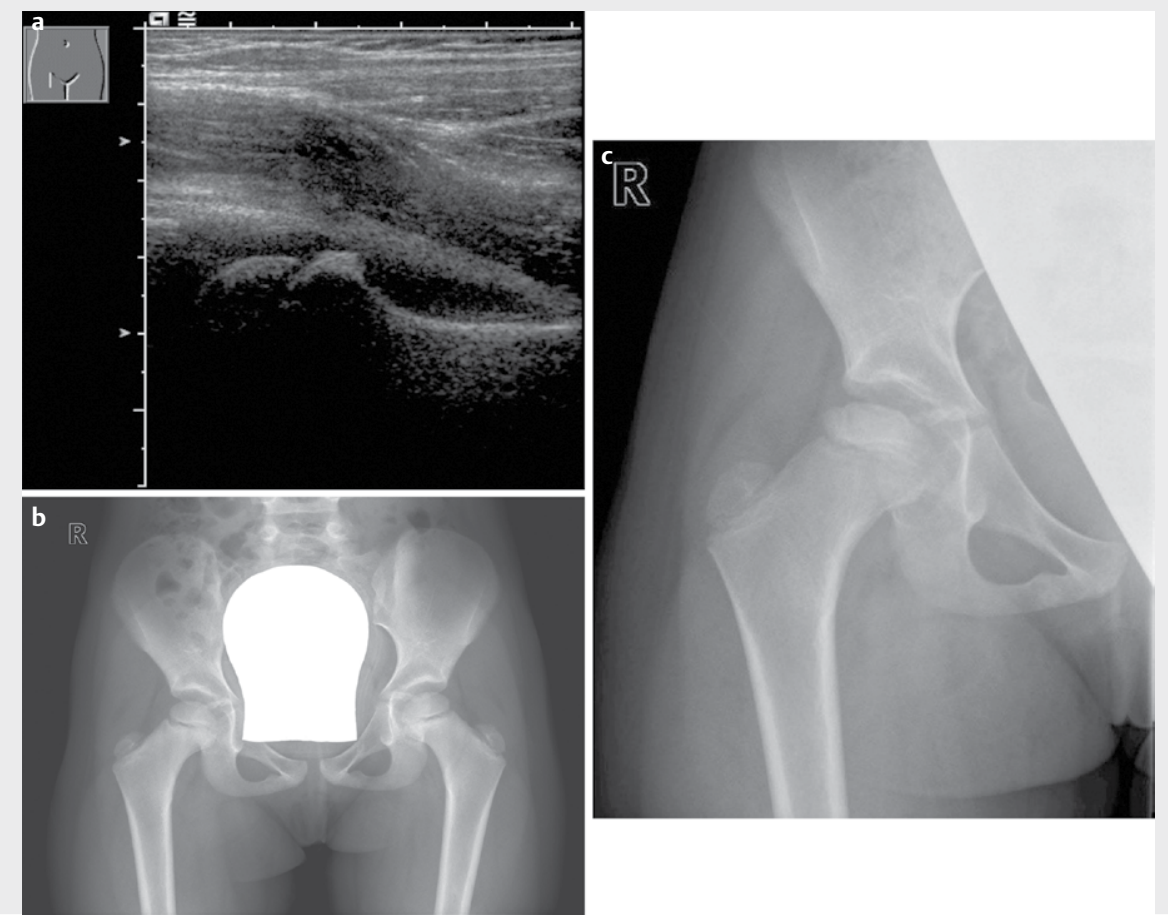

- Abb. 2 a 7-jähriges Mädchen mit Verdacht auf eine Coxitis fugax rechts mit sonografischem Hüftgelenkserguss mit einer Tiefenausdehnung von $3,7 \mathrm{~mm}$ im ventralen Longitudinalschnitt. b Normale Röntgenübersichtsaufnahme des Beckens bei Erstpräsentation mit klinisch eingeschränkter Hüftbeweglichkeit bei gutem Allgemeinzustand. c Radiologische Veränderungen im Sinne eines Morbus Perthes der rechten Hüfte bei derselben Patientin im Röntgenbild nach 3 Monaten. Die Patientin hatte im Intervall intermittierende Schmerzen im rechten Hüftgelenk.

Coxitis fugax von einigen Autoren mit einem Anteil von bis zu $17 \%$ angegeben, wobei spätere Untersuchungen einen ätiopathogenetischen Zusammenhang nicht bestätigt haben [5, 9, 13]. Fest steht, dass sich der Morbus Perthes im Anfangsstadium genau gleich präsentieren kann wie die Coxitis fugax (nur Gelenkerguss im Ultraschall ohne konventionell radiologische Veränderungen) und erst im Laufe der Monate pathognomonische Zeichen im Röntgen sichtbar werden. Deshalb findet man bis heute in der Literatur zu einer routinemäßigen Nachkontrolle nach einer Coxitis fugax unterschiedliche Empfehlungen: so gibt es Autoren, die keine Notwendigkeit für eine routinemäßige radiologische Nachkontrolle sehen $[9,13]$, andere sprechen sich eindeutig dafür aus [18].

In unserer Klinik bekam bis zur Auswertung der Studie jedes Kind, das mit einer Coxitis fugax behandelt und nachkontrolliert wurde, ungefähr 3 Monate nach Abheilung eine klinische und radiologische routinemäßige Nachkontrolle (in der Regel Hüftröntgen a.p. und Lauenstein-Aufnahme), um auf keinen Fall eine aseptische Hüftkopfnekrose zu verpassen, und gegebenenfalls frühzeitig intervenieren zu können.

Die vorgestellte Arbeit befasst sich mit der Wertigkeit der Röntgenverlaufskontrolle nach einer Coxitis-Episode in der Früherkennung eines Morbus Perthes. Ziel war es, an unserem Patientengut die Frage zu beantworten, ob die Röntgenkontrolle nach 3 Monaten routinemäßig notwendig ist oder ob es ausreicht, ein Röntgenbild nur bei Kindern zu machen, die im klinischen Verlauf zwischen Abheilung einer vermuteten Coxitis fugax-Episode und dem Kontrolltermin nach 3 Monaten andauernde oder intermittierende Beschwerden haben. Dies unter der Annahme, dass all diejenigen Kin- der, welche später einen Morbus Perthes entwickeln und sich im Frühstadium mit einer von der Coxitis fugax nicht zu unterscheidenden Symptomatik präsentieren, im klinischen Verlauf weiterhin Beschwerden haben, und somit die Indikation für weitere Diagnostik in diesen Fällen gegeben ist.

Die Bestätigung der Arbeitshypothese würde so einen Beitrag zur Senkung der Strahlenbelastung bei Kindern leisten können und unnötige Strahlenbelastungen der Genitalregion verhindern. Die Minimierung der Belastung mit ionisierender Strahlung spielt im Kindesalter eine wichtige Rolle, da das kindliche Gewebe besonders strahlenempfindlich ist und somit ein erhöhtes strahlenbedingtes Entartungsrisiko besteht. Zudem haben sie als potenzielle Eltern ein genetisches Strahlenrisiko [1]. Wo immer möglich sollte deshalb auf eine unnötige Strahlenbelastung verzichtet werden, ohne dabei auf die diagnostische Sicherheit zu verzichten.

Zusätzlich würden sich durch das Vermeiden von unnötigen Röntgenbildern auch Kosten und Aufwand für die Nachkontrolle reduzieren.

\section{Patienten und Methode}

In der vorgestellten Studie wurden retrospektiv alle Kinder und ihre Röntgenbilder, die in unserer Klinik zwischen 2004 und 2010 mit der Diagnose einer Coxitis fugax oder eines Morbus Perthes behandelt wurden, analysiert. Die Studie wurde bewilligt durch die kantonale Ethikkommission. Eine Einverständniserklärung der Patienten respektive der Eltern für diese retrospektive Studie mit anonymisierten Patientendaten war nicht notwendig. Die notwendigen Patienteninformationen wurden aus der digitalen Röntgendaten- 
bank (EasyVisionPACS), aus dem digitalen Patientendossier oder dem Krankengeschichtenarchiv entnommen.

Bei den Kindern mit der Diagnose einer Coxitis fugax wurden aus den vorhandenen Akten folgende Daten erhoben: Geschlecht, Alter des Kindes bei Diagnosestellung, Symptomatik, betroffene Hüftseite, Ultraschallbefund bei Erstkonsultation, konventioneller Röntgenbefund bei Erstkonsultation (Beckenübersicht und Lauenstein-Aufnahme der betroffenen Seite), klinischer Verlauf nach Abheilung, Röntgenbefund in der Kontrolle nach 3 Monaten (Hüftröntgen a.p. und Lauenstein-Aufnahme der betroffenen Seite), exakter Zeitpunkt des Verlaufsröntgen, sowie allenfalls spätere Diagnosestellung einer aseptischen Hüftkopfnekrose.

Es wurden 212 Kinder mit einer diagnostizierten Coxitis fugaxEpisode im vorgegebenen Zeitraum identifiziert. Bei 14 Kindern fehlte das für diese Studie essenzielle Verlaufskontrollröntgenbild, diese Patienten wurden aus der Studie ausgeschlossen. Somit wurden 198 Patienten für diese Studie berücksichtigt.

Die Sensitivität und Spezifität sowie die positiven und negativen prädiktiven Werte der Röntgenuntersuchung in der Diagnose eines Morbus Perthes wurden berechnet:

Sensitivität $=(\mathrm{N}$ richtig positiver $) /(\mathrm{N}$ richtig positive $+\mathrm{N}$ falsch negativer)

Spezifität $=(\mathrm{N}$ richtig negativer $) /(\mathrm{N}$ richtig negativer $+\mathrm{N}$ falsch positiver)

Positiver prädiktiver Wert $=r p /(r p+f p)$

Negativer prädiktiver Wert $=r n /(r n+f n)$

$\mathrm{rp}=$ richtig positive; $\mathrm{rn}=$ richtig negative; $\mathrm{fn}$ = falsch negative; $\mathrm{fp}=$ falsch positive.

Die statistische Aufarbeitung der Daten wurde mittels SAS 9.2 (SAS Institute Inc, Cary, NC, USA) durchgeführt.

\section{Ergebnisse}

Von den 198 Patienten mit der initialen Verdachtsdiagnose einer Coxitis fugax waren 135 männlich und 63 weiblich.

Das Durchschnittsalter der eingeschlossenen Patienten betrug 6,5 Jahre (1-14 Jahre).

22 Kinder waren jünger als 4 Jahre und 14 Kinder älter als 10 Jahre.

Beide Hüftgelenke wurden in etwa gleich häufig befallen. Das rechte Hüftgelenk war in 105 Fällen betroffen, das Linke in 89 Fällen. Bei 4 Patienten waren die Symptome bilateral.

Bei 180 Patienten wurden bei Erstkonsultation sowohl eine Ultraschalluntersuchung des Hüftgelenks, wie auch ein Röntgenbild gemacht. Bei 12 Patienten fand sich nur ein Ultraschall, ohne Röntgenbild. Bei 5 Patienten nur eine Röntgenuntersuchung ohne Ultraschall und bei 1 Patienten fand sich weder ein Ultraschall noch ein konventionelles Röntgenbild der Hüfte in unserem Archiv.

Die Ultraschalluntersuchung zeigte bei 179 Patienten einen einseitigen Erguss, bei 2 Patienten einen Erguss beidseits, bei 1 Patienten ödematöse Weichteile, bei 1 Patienten eine verdickte Synovia, bei 1 Patienten einen Erguss und eine verdickte Synovia und bei 8 Patienten keinen Erguss. In der Beckenübersicht und der Lauenstein-Aufnahme der betroffenen Seite bei Erstkonsultation war bei 5 Patienten ein beginnender Morbus Perthes nicht auszuschließen, bei den restlichen 180 Patienten mit vorhandenem Röntgen- bild war der Röntgenbefund unauffällig, insbesondere ohne Hinweise auf eine aseptische Hüftkopfnekrose.

Die radiologische Nachkontrolle des betroffenen Hüftgelenks fand bei 192 Patienten zwischen 1-6 Monate nach Coxitis-Episode statt, davon bei 184 Patienten exakt nach 3 Monaten. Bei 1 Patienten wurde wegen seines Alters, welches oberhalb der hauptsächlichen Altersinzidenz für Coxitis fugax lag, und seiner starken, persistierenden Beschwerden nach 4 Monaten ein MRI gemacht, welches keine Hinweise für eine aseptische Hüftkopfnekrose zeigte.

Im Verlauf zwischen Diagnosestellung und radiologischer Nachkontrolle hatten 20 Patienten rezidivierende oder persistierende Hüftbeschwerden wie Hinken oder Schmerzen. Davon hatten 16 Patienten eine unauffällige radiologische Verlaufsbildgebung, bei 4 Patienten wurde ein Morbus Perthes diagnostiziert (Sensitivität 0,2). Bei keinem Patient, der im Verlauf zwischen Coxitis fugax-Episode und dem Kontrollröntgenbild keine Beschwerden hatten, wurde im Verlaufsröntgenbild ein Morbus Perthes diagnostiziert (negativer prädiktiver Wert: 0,92).

Von den 5 Patienten, bei denen sich im Röntgen bei Erstkonsultation Hinweise auf eine aseptische Nekrose gezeigt hatten, wurde bei 1 Patienten später definitiv ein Morbus Perthes diagnostiziert. Bei den anderen 4 konnten die Hinweise später nicht mehr bestätigt werden.

Von den 198 Patienten, mit einer im Zeitraum von 2004 bis 2010 aufgetretenen fraglichen Coxitis-Episode, wurde also schlussendlich bei insgesamt 4 Patienten ein Morbus Perthes diagnostiziert (richtig positiv: 4). Jeder dieser 4 Patienten zeigte im klinischen Verlauf rezidivierende oder persistierende Hüftbeschwerden nach Erstkonsultation (positiver prädiktiver Wert: 1,0, Spezifität: 1,0). Von den 4 Patienten hatten 3 bei Erstkonsultation ein unauffälliges Röntgenbild, bei 1 war das Röntgenbild suspekt auf einen Morbus Perthes, ohne das aber im initialen Röntgenbild eindeutig die Diagnose eines Morbus Perthes gestellt werden konnte.

Außerdem war bei einem Patienten mit einer vermuteten Coxitis fugax-Episode, der nicht zur klinischen und radiologischen Nachkontrolle nach 3 Monaten erschienen war, 1,5 Jahre nach CoxitisEpisode im Röntgen ein Morbus Perthes zu sehen. Der Patient hatte nach der Coxitis-Episode ein persistierendes Schonhinken gezeigt.

\section{Diskussion}

Das vorrangige Ziel dieser Arbeit war es, herauszufinden, ob sich jedes Kind mit einer vermuteten Coxitis fugax-Episode als Verlaufskontrolle 3 Monate nach Erstdiagnose der Coxitis fugax konventionellen Röntgenbildern unterziehen muss, um keinesfalls eine aseptische Hüftkopfnekrose zu verpassen, oder ob dies nur bei denjenigen Kindern indiziert ist, die im klinischen Verlauf und bei der gezielten klinischen Untersuchung nach 3 Monaten persistierende oder rezidivierende Hüftbeschwerden haben. Das regelmäßige Durchführen einer Röntgenkontrolle 3 Monate nach einer vermuteten Coxitis fugax wurde in unserer Klinik zu einer Zeit eingeführt, als es noch keine hochauflösenden Ultraschall-Geräte oder MRTs gab und hat sich, wie so vieles in der Medizin, über mehrere Jahrzehnte gehalten.

Die Ergebnisse unserer retrospektiven Studie zeigen, dass alle Kinder mit einer durchgemachten Coxitis-Episode, bei denen sich später ein Morbus Perthes entwickelte (d. h. die Coxitis-Episode im 
Nachhinein als Erstmanifestation eines Morbus Perthes zu interpretieren ist), im klinischen Verlauf weiterhin rezidivierende oder persistierende Hüftbeschwerden hatten (positiv prädiktiver Wert: 1,0, Spezifität: 1,0$)$.

Wir konnten in unserer retrospektiven Analyse der Patienten kein Kind identifizieren, das nach einer Coxitis-Episode im Verlauf 3 Monate beschwerdefrei und klinisch unauffällig war und dann in der Röntgenkontrolle einen Morbus Perthes zeigte.

In verschiedenen bereits publizierten Studien wurden unterschiedliche Zeitpunkte für die Nachkontrolle nach einer Coxitis fugax-Episode genannt. Eine aktuelle Übersichtsarbeit von Asche et al. empfiehlt, nach einer Coxitis fugax-Episode während mindestens 6 Monaten Nachkontrollen durchzuführen [2]. In den eingeschlossenen Studien dieser Übersichtsarbeit sieht man, dass grundsätzlich bei denjenigen Kindern ein Perthes diagnostiziert wird, bei welchen die Coxitis fugax-Episode atypisch verläuft, d. h. mit persistierenden oder intermittierenden Beschwerden über den normalen Verlauf einer Coxitis fugax hinaus. Dies deckt sich mit den Ergebnissen unserer Studie. Gemäß den Resultaten unserer Studie erscheint es ausreichend, eine klinische Nachkontrolle nach 3 Monaten durchzuführen, um einen allfälligen Morbus Perthes bei den Kinder mit der Verdachtsdiagnose einer Coxitis fugax nicht zu verpassen. Bis dato konnte kein eindeutiger Zusammenhang zwischen einer Coxitis fugax und der Entstehung eines Morbus Perthes nachgewiesen werden $[13,15,20]$. Stobbe et al. konnte zwar eine statistisch erhöhte Inzidenz eines Morbus Perthes bei Jungen nach durchgemachter Coxitis fugax zeigen, eine eindeutige Prädisposition der Coxitis fugax für das Auftreten eines Morbus Perthes konnte aber nicht bewiesen werden [16]. Umso wichtiger ist die ausführliche Information der Eltern über das Krankheitsbild des Morbus Perthes, um über die Notwendigkeit einer erneuten Konsultation bei Auftreten von Beschwerden erst nach der Nachkontrolle nach 3 Monaten aufmerksam zu machen.

Die vorliegende Arbeit hat gewisse Limitationen. Diese begründen sich vor allem im retrospektiven Studiendesign mit den dadurch bedingten Nachteilen und der Tatsache, dass es sich um eine Erhebung an nur einem Zentrum handelt. Zudem ist die Rate der „Events“, d. h. des Auftretens eines Morbus Perthes, sehr niedrig, was ebenfalls dazu führt, dass die dargestellten Ergebnisse mit einer gewissen Vorsicht zu interpretieren sind.

\section{Schlussfolgerung}

Aufgrund der Resultate der dargelegten Studie konnten wir unsere Annahme bestätigen, dass ein Verlaufsröntgenbild zum Ausschluss eines Morbus Perthes nach einer vermuteten Coxitis fugax-Episode nur dann notwendig ist, wenn im klinischen Verlauf und in der

Hüftgelenksuntersuchung weiterhin persistierende oder rezidivierende Beschwerden auftreten. In unserem Patientengut von 198 Patienten hätte man somit bei 178 Patienten, die im Verlauf beschwerdefrei waren, auf ein konventionelles Kontrollröntgen verzichten können. Dadurch hätte man einerseits einen Beitrag zur Reduktion von Röntgen bei Kindern im Wachstumsalter geleistet, andererseits könnten auch die Kosten und der Aufwand für die häufigen Coxitis-Nachkontrollen gesenkt werden. Bei persistierenden oder rezidivierenden Hüftbeschwerden nach einer Coxitis fugax ist es weiterhin unabdingbar, konsequent weitere diagnostische Abklärungen durchzuführen.

Unsere Empfehlung für die Nachkontrolle eines Patienten mit Coxitis fugax ist daher eine

klinische Nachkontrolle bei einem Spezialisten (pädiatrischer Orthopäde/Kinderchirurg)

3 Monate nach vermuteter Coxitis-Episode mit genauer Verlaufsanamnese, klinischer

Untersuchung und ausführlicher Instruktion des Patienten respektive der Eltern, bei erneutem Auftreten von Beschwerden wieder vorstellig zu werden.

\section{Stellungnahme zur Autorenschaft}

U. Lenoir: Konzeptentwicklung, Erhebung der Patientendaten. Datenanalyse/Interpretation, Verfassen des Manuskripts.T. Slongo: Behandlung eines Grossteils der Patienten, Hilfe bei der Konzeptentwicklung, kritischer Review der Analysedaten, Review des Manuskripts.E. Aghayev: Analyse und statistische Berechnung der Daten, Manuskript Review.A. Joeris: Idee und Hilfe bei der Konzeptentwicklung für die Studie. Hilfestellung bei der Interpretation der analysierten Daten. Kritischer Review des Manuskripts.

\section{Danksagung}

Wir danken Dr. med. Rainer Wolf, Leiter Kinderradiologie am Universitätsspital für Diagnostische, Interventionelle und Pädiatrische Radiologie, Inselspital, Universitätsspital Bern, Schweiz, für die zur Verfügung gestellten Röntgen-und Ultraschallbilder sowie für die Unterstützung bei der Bildverarbeitung.

Interessenkonflikt

Die Autoren erklären hiermit, dass kein Interessenkonflikt besteht.

\section{Literatur}

[1] Alzen G, Benz-Bohm G. Radiation protection in pediatric radiology. Dtsch Arztebl Int 2011; 108: 407-414 Epub 2011 Jun 17

[2] Asche et al. What is the clinical course of transient synovitis in children: a systematic review of the literature. Chiropractic \& Manual Therapies 2013; 21: 39

[3] Bernd L, Niethard FU, Graf J et al. The transient coxitis fugax. Z Orthop 1992; 130: 529-535

[4] Bickerstaff DR, Neal LM, Booth AJ et al. Ultrasound examination of the irritable hip. J Bone Joint Surg Br 1990; 72: 549-553

[5] de Pellegrin M, Fracassetti D, Ciampi P. Coxitis fugax. The role of diagnostic imaging. Orthopade 1997; 26: 858-867 [Article in German]

[6] Fabry G. Clinical practice: the hip from birth to adolescence. Eur ] Pediatr 2010; 169: 143-148

[7] Gledhill RB, McIntyre JM. Transient synovitis and Legg-Clavé-Perthes Disease: a comparative study. Can Med Assoc J 1969; 100: 311-320

[8] Graf J, Bernd L, Niethard FU et al. Diagnosis of coxitis fugax, the most frequent hip disease in childhood. Klin Padiatr 1991; 203: 448-451 
[9] Kallio P, Ryöppy S, Kunnamo I. Transient synovitis and Perthes' disease. Is there an aetiological connection? J Bone Joint Surg Br 1986; 68808-68811

[10] Kim HK, Herring JA. Pathophysiology, classifications, and natural history of Perthes disease. Orthop Clin North Am 2011; 42: 285-295

[11] Konermann W, de Pellegrin M. The differential diagnosis of juvenile hip pain in the ultrasonographic picture. Transient coxitis. Legg-CalvéPerthes disease, epiphysiolysis of the femur head. Orthopade 1993; 22: $280-287$

[12] Kramer J, Hofmann S, Scheurecker A et al. Perthes disease. Radiologe 2002; 42: 432-439 [Article in German]

[13] Landin LA, Danielsson LG, Wattsgård C. Transient synovitis of the hip. Its incidence, epidemiology and relation to Perthes' disease. J Bone Joint Surg Br 1987; 69: 238-242

[14] Moens P, Fabry G. Legg-Calvé-Perthes disease: one century later. Acta Orthop Belg 2003; 69: 97-103
[15] Mumme T, Berkemeier E, Maus U et al. Coxitis fugax - the beginning of Perthes' disease? Z Orthop Ihre Grenzgeb 2005; 143: 529-533

[16] Stobbe S, Pennekamp PH, Filler T et al. Does Coxitis Fugax Predispose for Later Perthes' Disease? - First Results of an Insurance Data-Based Study. Z Orthop Unfall 2015; 153: 80-84

[17] Taylor GR, Clarke NM. Management of irritable hip: a review of hospital admission policy. Arch Dis Child 1994; 71: 59-63

[18] Waters E. Toxic synovitis of the hip in children. Nurse Pract 1995; 20: 44-46, 48, 51

[19] Wenger DR, Ward WT, Herring JA. Legg-Calvé-Perthes disease. J Bone Joint Surg Am 1991; 73: 778-88

[20] Wirth T. Coxitis fugax - the beginning of Perthes' disease? Z Orthop Ihre Grenzgeb 2006; 14: 232 